\title{
Das Leben in Szene setzen
}

\section{Wege zu einer relationellen Sprachdramaturgie}

\section{Daniel Feldhendler}

\begin{abstract}
Zusammenfassung
Nach einem ersten Beitrag in Scenario 2, 2007 mit dem Titel „Playback Theatre: A Method for Intercultural Dialogue“ stellt nun der Autor im ersten Teil dieses Artikels seinen spezifischen Ansatz vor. Ab 1977 entwickelte Daniel Feldhendler gemeinsam mit Marie und Bernard Dufeu pädagogische Verfahren für den Fremdsprachenunterricht, die von Psychodrama und Dramapädagogik stark geprägt wurden. Im zweiten Teil seines Beitrages veranschaulicht der Autor, wie er Playback Theater gezielt in seinen Lehrveranstaltungen in der Ausbildung von Studierenden am Institut für Romanische Sprachen und Literaturen der J.W. GoetheUniversität in Frankfurt am Main einsetzt. Hauptziele dabei sind: Entwicklung von Sprachkompetenz durch globale Lernaktivierung, Abbau von Lern- und Sprechhemmungen, Aufbau einer kommunikativen Atmosphäre, Förderung der Autonomie, erfahrungsbezogenes Lernen durch Handeln und durch Mitteilung biografischer, persönlicher Erlebnisse, Vermitteln innovativer Arbeitsansätze in der Lehre an der Hochschule.
\end{abstract}

\section{Einleitung}

Seit 1977 wurden in Deutschland pädagogische Verfahren für den Fremdsprachenunterricht entwickelt, die von Psychodrama und weiteren ganzheitlichen humanistischen Verfahren in Verbindung mit Grundlagen der Dramapädagogik stark geprägt sind. Von diesem Zeitpunkt an begann eine Phase schöpferischer Gestaltung am gemeinsam gegründeten Centre de Psychodramaturgie in Mainz (Gründer: Marie und Bernard Dufeu, Daniel Feldhendler). Während Marie und Bernard Dufeu vor allem die Methode der „Psychodramaturgie Linguistique $(P D L)$ " und Grundlagen einer Relationellen Pädagogik entwickelten, konzentrierte ich mich vorrangig auf den Aufbau einer „Dramaturgie relationnelle“ (Relationelle Dramaturgie) an der Goethe-Universität in Frankfurt am Main. 


\section{Eine Relationelle Dramaturgie}

Geprägt einerseits durch die gemeinsame Aktionsforschung mit Marie und Bernard Dufeu bei der praktischen Anwendung der PDL und andererseits durch die intensive Beschäftigung mit den Verfahren der Spiel-, Interaktions- und Theaterpädagogik (Fortbildungserfahrungen mit französischen Ansätzen der Theaterpädagogik, Ausbildung bei und später Zusammenarbeit mit Augusto Boal und Jonathan Fox), wandte ich mich ab 1981 verstärkt der Entwicklung einer Relationellen Dramaturgie zu.

Als ganzheitliche Arbeitsweise im Fremdsprachenunterricht verbindet sie Psychodrama und Dramaturgie (siehe Bibliographie).

Psychodramatisch zu arbeiten bedeutet, eine zwischenmenschliche Begegnung herbeizuführen, die durch gegenseitige Empathie geprägt ist: authentische Ausdruckswünsche der Adressaten sollten dabei im Vordergrund des Geschehens stehen. Wenn psychodramatische Methoden im Fremdsprachenunterricht eingesetzt werden, bedeutet dies, dass Begegnung, also die Interaktion der Teilnehmer (TN), ihre Beziehung zu sich selbst, zueinander und zum weiteren Umfeld, die Ausgangspunkte für Sprechanlässe bilden. Die Dynamik des Prozesses, innerhalb dessen die Fremdsprache erworben wird, basiert nicht auf vorgegebenen Übungen aus Lehrbüchern, sondern auf der Situation im Hier und Jetzt und auf der Beziehungsebene zwischen den TN und dem Geschehen in der Lerngruppe (vgl. http://www.psychodramaturgie.de). Dabei wird das Setting sowohl durch relationelle als auch dramaturgische Arbeitsformen getragen. Relation meint: in Beziehung setzen. Relationelles Lehren geht daher von den Situationen der Lernenden selbst aus. Als Interaktion ist es somit auf die einzelnen TN in der Lerngruppe zentriert.

Der Lernprozess ist an einer relationellen Progression orientiert (Ausdruckswünsche und -bedürfnisse von TN in einem bestimmten Kontext). Auch den linguistischen Anforderungen, die den Lernprozess begleiten, wird in diesen Arbeitsformen Rechnung getragen.

In der Relationellen Dramaturgie wie in der Sprachpsychodramaturgie wird auch das Psychodrama mit seinen Prinzipien und Strukturen umgesetzt. Zu den gängigen Techniken (Doppeln, Spiegel, Rollenwechsel, Rollentausch, innerer Monolog, Interviewtechniken usw.) und dramapädagogischen Arbeitsformen (wie Skulpturen, Vignetten als kurze Szenen bzw. Rollenspiele) kommen Arbeitsformen aus der weiteren Tradition des Psychodramas. Sein Begründer, J.L. Moreno (1889-1974) sprach von action methods - gemeint sind Stegreiftheater, Lebende Zeitung, Soziodrama, Axiodrama, Ethnodrama, Soziometrie, Rollenspiel und weitere Psychodrama-Methoden, die die "schöpferische Spontaneität" wecken und fördern sollen (vgl. http://www . psychodrama-deutschland.de/).

Die hier erwähnten weiteren psychodramatischen Arbeitsformen sind auch für interkulturelle Lernprozesse besonders geeignet. In den psychodramatischen Lernprozessen ist eine Dimension, die ich als Einübung in Intersubjektivität bezeichne: es umschreibt die aktive Beschäftigung mit der Selbst- und Fremdwahrnehmung der Lernenden bzw. Teilnehmer von Fortbildungen durch die 
Darstellung der Selbst- und Fremdbilder in der interkulturellen Begegnung. Perspektiven und Standpunkte werden im eigentlichen und übertragenen Sinne gewechselt. Dies wird wortwörtlich in Raum und Zeit im Rollentausch handelnd umgesetzt. Durch die psycho- und soziodramatischen Mittel werden die verschiedenen Wahrnehmungsebenen und die unterschiedlichen Standpunkte hervorgehoben, so dass das interkulturelle Feld in seiner Komplexität erschlossen und die Dynamik des interkulturellen Prozesses erlebnis- und erfahrungsorientiert vermittelt werden kann.

Durch diese soziodramatische Bewusstmachung und Erweiterung der Selbstund Fremdwahrnehmung wird ein aktiver Zugang sowohl zu den Stereotypen, zu den Vorurteilen im Sinne von Fehlurteilen, wie auch zu den subjektiven Bildern, Vorstellungen und Erlebnissen möglich. Es entsteht ein gemeinsamer Austausch in der Zielsprache über die Wertvorstellungen (Axiodrama) und die verbindenden Erfahrungen in den erlebten Begegnungen mit dem Anderen, mit dem Fremden.

In der Relationellen Dramaturgie ist der Begriff Dramaturgie vom Begriff Drama abgeleitet, der etymologisch auf Handlung verweist. Dramaturgie beinhaltet sowohl die äußere Form der Handlung als auch die innere Struktur, die das Handlungsgeschehen beeinflusst und bestimmt. Die Dramaturgie ist die notwendige Form zur Bildung einer 'Stegreiflage' und 'Stegreifeignung' im Sinne Morenos. Die Dramaturgie bildet also das katalysierende Gerüst für die Handlung, indem sie im Fremdsprachenunterricht durch die Struktur der offenen Rahmenhandlungen vermehrt Anlässe zum sprachlichen Ausdruck bietet. Sie fördert den Lernprozess, wobei verbaler und nichtverbaler Ausdruck in eine ganzheitliche Arbeitsform integriert sind. Dramaturgie wird hier als organisierendes Prinzip für die Stegreifarbeit und als Förderinstrument für den spontanen, authentischen und persönlichen Ausdruck aus dem Erleben verstanden.

\section{Die Arbeitsstrukturen der relationellen Dramaturgie}

Die Relationelle Dramaturgie fördert die Lernaktivität und die Kreativität von Teilnehmern im Fremdsprachenunterricht. Dieses Verfahren kann in Gruppen unterschiedlicher Größe als Intensivform (Blockveranstaltung) oder im herkömmlichen Unterricht eingesetzt werden:

- zur Unterstützung und als Begleitform in den ersten Stunden eines traditionellen Anfängerunterrichts;

- als eigenständige Methode bei fortgeschrittenen Teilnehmern.

Durch die handlungs- und erfahrungsorientierte Pädagogik erleben die Teilnehmer in speziellen Aufwärm- und Interaktionsübungen, Phantasie- und Stegreifübungen einen intensiven und situationsgerechten Zugang zur Fremdsprache. Analog dem psychodramatischen Modell gliedert sich der Unterrichtsverlauf in der Regel in drei Phasen: 
- Aufwärm- und Sensibilisierungsphase;

- Bearbeitungs- und Vertiefungsphase;

- Integrations- und Abschlussphase.

\subsection{Aufwärm- und Sensibilisierungsphase}

Jede Unterrichtssequenz beginnt deshalb mit dieser Phase, weil der Einstieg in die Arbeit richtungsweisend für die spätere Qualität der Begegnung und des Austausches ist. Er bereitet den Boden für die weitere Lernatmosphäre der Gruppe und legt einen Grundstein für die Bereitschaft der Lernenden zur Öffnung, Vertrauensbildung und Zusammenarbeit. Die Übungen in dieser Phase sind sehr strukturiert und werden vom Lehrer bzw. Trainer angeleitet. Sie gehen oftmals von den nichtverbalen Ausdrucksformen aus und führen zum verbalen Ausdruck hin.

In dieser Phase werden Arbeitsformen eingesetzt wie:

- Atem- und Entspannungsübungen;

- Visualisierungsübungen;

- Übungen zu Raum und Zeit;

- Rhythmusübungen;

- Bewegungs- und Interaktionsübungen.

Diese relationellen Übungen geben den TN die Möglichkeit sich zu sammeln, "warm" zu werden mit der fremden Sprache. Sie fördern erste Kontaktaufnahme mit anderen TN und mit der gesamten Gruppe. Der strukturierte Aufbau der Übungen leitet zur gruppenzentrierten bzw. themenzentrierten Orientierung der Sprachvermittlung hin und sensibilisiert die TN für die weitere Bearbeitung.

\subsection{Bearbeitungs- und Vertiefungsphase}

Hier werden die Situationen und Themen aktiv umgesetzt. Die TN lernen paralinguistische Elemente in der Handlung zu beherrschen. Spontaneität und Wahrnehmung unterstützen die Kommunikation. Sprechen und Handeln als Mittel der Kommunikation geschehen in der Hier- und- Jetzt- Situation. Der Ort des Szenischen beinhaltet einen schöpferischen Spannungsbereich und stellt somit einen "potenziellen Raum" dar, in dem die sprachliche bzw. kulturelle Produktion stattfindet. Die Situationen spielen auf den Ebenen des Realen, Imaginären und Symbolischen. Indem sich die Teilnehmer stärker einbringen (als im herkömmlichen Fremdsprachenunterricht) und sich mit einer Rolle identifizieren, wird die Sprache in einem unmittelbaren Zusammenhang erlebt.

In dieser Phase werden Aktivitäten mit offenem Handlungsrahmen eingesetzt wie: 
- dramaturgische und interaktionelle Übungen mit verschiedenen Mitteln als Übergangsobjekte wie Fotos, Bilder, Postkarten, Zeichnungen, Gegenstände, Handpuppen, Einsatz von leeren Stühlen;

- dramaturgische und relationelle Bearbeitung von Texten (alle Textgattungen: Gedichte, Chansons, Zeitungsartikel, Kurzgeschichten, Märchen, Romane, Mythen);

- szenischeUmsetzung zur Dynamisierung der Handlung und zurFörderung der Lernprozesse;

- psychodramatische Szenen und Rollenspiele zur Vertiefung;

- elaborierte dramaturgische Formen wie z.B. Stegreiftheater, Bildertheater, Lebendiges Zeitungstheater, Playback Theater zur Darstellung alltäglicher Situationen, Forum-Theater als dialogisches Prinzip;

- soziodramatische Übungen (z.B. Rollenspiele als social drama zur Behandlung kultureller, gesellschaftlicher Themen bzw. zur Förderung der interkulturellen Selbst- und Fremdwahrnehmung durch Übernahme von sozialen Rollen).

In dieser Phase werden auch mündliche und schriftliche Aktivitäten integriert. Wir bevorzugen relationelle Arbeitsformen in Verbindung mit Prinzipien des kreativen Schreibens.

\subsection{Integrations- und Abschlussphase}

Diese Phase dient zunächst dazu, den intermediären Raum des Szenischen zu verlassen. Es ist die Zeit, zurückzukehren zum realen Raum. Rollen werden durch symbolische Übungen abgelegt. Auch strukturierte Rollenrückmeldungen in der Fremdsprache können hier stattfinden. Je nach Situationen wird das Prinzip des Sharing umgesetzt und erweitert: das persönliche Mitteilen dient dazu, gemeinsame Erfahrungen und deren Resonanz zu verbinden. Metakommunikative Äußerungen in der Fremdsprache wie z.B. Formen der Rückmeldung (aus dem Erleben, zur Sitzung etc.) werden ebenfalls integriert. Diese Phase ist auch der Moment für eine vertiefte Aufarbeitung linguistischer Fragen (Grammatik, Semantik, Wortschatz etc.), die während des Verlaufs aufgetreten sind.

\section{Aus der Praxis: Ansätze mit Playback Theater}

\subsection{Ein interaktives Theater}

Ab 1975 entwickelte Jonathan Fox gemeinsam mit seiner Lebenspartnerin Jo Salas und mit ihrer Truppe in den USA (New York State) eine besondere 
Form des interaktiven Theaters. Von seinen Begründern Playback Theatre (PT) genannt, kennzeichnet es soziale Kommunikation mit ästhetischen Mitteln. Es ist eine Improvisationsform, in welcher persönlich erlebte Erfahrungen und Erlebnisse von Zuschauern verbal mitgeteilt und von Schauspielern aus dem Stegreif auf die Bühne gebracht werden. Die Originalität der Methode beruht auf dieser spontanen szenischen Umsetzung in einer sehr dynamischen offenen Dramaturgie. PT setzt sich damit zum Ziel, den zwischenmenschlichen Dialog zu fördern und Menschen miteinander in Verbindung zu bringen. Es kann an jedem Ort und in jedem Raum aufgeführt werden. Eine feste Struktur ist als Rahmen vorgesehen, eine Art Ritual, das dazu dient, die persönlichen Erzählungen von Menschen auf eine achtsame Weise zu ermöglichen, empathisch und schöpferisch zu spiegeln und zu gemeinsamen Erfahrungen zu verknüpfen.

Die aktuelle Verbreitung dieser Methode in verschiedenen kulturellen Zusammenhängen auf allen Kontinenten der Welt lässt sich durch die Tatsache erklären, dass PT über seine ursprüngliche Funktion als Aufführungsform hinaus eine Form der unmittelbaren zwischenmenschlichen Begegnung mit hoher Flexibilität und vielfältigen Anwendungsmöglichkeiten darstellt. PT bietet durch sein narratives Prinzip einer aktualitätsbezogenen Erzählkultur einen kreativen Weg zu einer dynamischen Kulturanthropologie.

\subsection{Geschichtlicher Rückblick}

Jonathan Fox (*1943) versteht PT als eine Renaissance von Volkstheater. Darin haben ihn seine Studien an der Universität Harvard zur Tradition mündlicher Überlieferungen (Oral history) und zu Untersuchungen von Gemeinschaften und ihrer Wertesysteme wesentlich beeinflusst. Weitere Einflussbereiche waren das klassische Psychodrama, in dem er ab 1973 am Ursprungsort dieser Methode am Moreno-Institut in Beacon ausgebildet wurde, und das amerikanische Experimentaltheater der 60er Jahre - insbesondere Richard Schechner und sein Ansatz der Theateranthropologie. Jonathan Fox beschreibt die Wiederbelebung dieser vorschriftlichen oralen Tradition als ein Mittel zur Bildung kultureller Gemeinschaften: PT gilt als Community Theatre und ist damit ein Theater der sozialen Partizipation.

Zunächst wurde PT in den Vereinigten Staaten weiter entwickelt und kam ab 1980 nach Australien und Neuseeland und 1984 nach Japan. In den folgenden Jahren entstanden PT-Gruppen in Europa. Ab 1988 gewann PT im deutschsprachigen Raum durch regelmäßige Workshops von Fox eine immer stärkere Präsenz. Etwa zum selben Zeitpunkt wurde die Methode auch in Südamerika weiter vermittelt. 1990 wurde das International Playback Theatre Network (IPTN) zur Förderung des Austausches in dieser Theaterbewegung ins Leben gerufen. Der Newsletter Interplay des IPTN erscheint mehrsprachig und seit 2000 auch online im Internet. 1993 gründete Fox die School of Playback Theatre in den USA (in New Paltz, NY), mit Verbindungen in Europa. Zu Beginn des 21. Jahrhunderts wird PT weltweit auf allen Kontinenten in mehr als 50 Ländern praktiziert. 
Nach nun mehr als 30 Jahren betrachten die Begründer Fox und Salas im Rückblick das Playback Theater als eine gelungene Integration, als eine Schnittstelle zwischen ästhetischer Erfahrung, sozialer Interaktion und gesellschaftlichem Ritual zur Förderung des zwischenmenschlichen Dialogs. Damit wird die Vision von einem Theater wahr, das der heutigen Gesellschaft etwas von der integrativen sozialen Funktion des Geschichtenerzählens und ästhetischer Rituale früherer Zeiten zurück vermittelt.

\subsection{Grundformen des Playback Theaters}

Die klassische Aufführungsform beginnt mit einer interaktiven Aufwärmphase mit dem Publikum über kurze Darstellungsformen (z.B. fließende Skulpturen - Fluids). Danach werden von einzelnen Zuschauern Erlebnisse, Erfahrungen und erlebte Situationen mitgeteilt und unmittelbar - aus dem Stegreif von Schauspielern umgesetzt, in verdichteter Form dargestellt und sozusagen zurückgespielt (daher play-back). Ein Teil des Raumes wird zur Bühne, wo die Spieler dem Publikum gegenüber sitzen. Rechts von den Zuschauern, umgeben von Instrumenten, ist in der Regel ein Musiker. Links stehen 2 Stühle: einer ist für den Leiter (Conductor), der andere für den Erzähler (Story Teller) aus dem Publikum. Er berichtet, auf strukturierte Fragen des Leiters hin, von einem persönlichen Erlebnis. Nach diesem Interview übergibt der Leiter an die Spieler und Musiker. Die Geschichte wird mimisch, mit Bewegungen, Worten, Tüchern als Requisiten und Musik begleitet und so dargestellt, dass die Essenz der Erzählung bildhaft erlebbar wird. Nach dem Spiel wird die Geschichte in einer symbolischen Form an den Erzähler sozusagen zurückgegeben, und dieser kommentiert die spontan entstandene szenische Umsetzung. Angeregt von dieser ersten Erzählung folgen in der Regel weitere Erzähler, und ihre Geschichten verbinden sich zu einem thematischen roten Faden. Persönliche und individuelle Erfahrungen werden zu einem kollektiven Erlebnis, das in situ entsteht, wobei Sprache, Musik, szenische Metaphern und viele dramaturgische Formen (Pair, Chorus, Tableau, Story usw.) den gemeinschaftlichen Dialog unterstützen.

Indem biografische Momente erzählt und unmittelbar in einer dynamischen Spiegelung umgesetzt werden, wirkt das Mitgeteilte gleich einem Vergrößerungsglas als Mittel der Selbsterkenntnis. Die Schauspieler werden zum „Sprachrohr" für Einzelne und Gruppen. PT erweist sich somit als wesentliches Instrument der Identitätsarbeit: es ermöglicht und fördert die Bildung einer „narrativen Identität“" (Konzept des französischen Philosophen Paul Ricoeur). Die eigene Geschichte auf diese Weise zur Sprache zu bringen heißt, die eigene Geschichte neu zu entdecken, aber auch, gehört und gesehen zu werden. Der dialogische Wert besteht darin, die eigene gelebte Geschichte und erlebte Erfahrung mitzuteilen und in Kooperation mit Anderen im Hier und Jetzt wieder zum Leben zu erwecken. Die Szene auf der Bühne auf sich wirken zu lassen und auf eigene Realität zu überprüfen, verbessert Selbst- und Fremdverstehen.

Fox und Salas selbst greifen zur theoretischen Begründung dieser Sachverhalte 
auf Oliver Sacks zurück. Oliver Sacks, 1933 in London geboren, dann praktizierender Arzt und Professor für Neurologie in New York, unterstrich schon früh den identitätsbildenden Wert von erzählten Lebensgeschichten in seinem viel beachteten Buch Der Mann, der seine Frau mit einem Hut verwechselte (1987):

Jeder von uns hat eine Lebensgeschichte, eine Art innerer Erzählung, deren Gehalt und Kontinuität unser Leben ist. Man könnte sagen, dass jeder von uns eine ,Geschichte' konstruiert und lebt. Diese Geschichte sind wir selbst, sie ist unsere Identität. Wenn wir etwas über jemanden erfahren wollen, fragen wir: Wie lautet seine Geschichte, seine wirkliche, innerste Geschichte? Biologisch und physiologisch unterscheiden wir uns nicht sehr voneinander - historisch jedoch, als gelebte Erzählung, ist jeder von uns einzigartig. Um wir selbst zu sein, müssen wir uns selbst haben; wir müssen unsere Lebensgeschichte besitzen oder sie, wenn nötig, wieder in Besitz nehmen. Wir müssen uns erinnern - an unsere innere Geschichte, an uns selbst. Der Mensch braucht eine solche fortlaufende innere Geschichte, um sich seine Identität, sein Selbst zu bewahren.

\subsection{Playback Theater an der Hochschule}

Seit Anfang 1990 setze ich das Playback Theater gezielt in Lehrveranstaltungen ein. Als Instrument der Innovation in alternativen Lehr- und Lernformen dient es integrierter Fremdsprachenausbildung (Zielsprache Französisch):

- im Grund- und Hauptstudium der Studierenden;

- in Theorie und Praxis der Fremdsprachendidaktik;

- in der Lehrerfortbildung (Schulen und Erwachsenenbildung).

In diesem pädagogischen Kontext dient Playback Theater einem aktiven Training der Reflexivität, als Form erweiterter Selbst- und Fremdwahrnehmung in der Kommunikation. Grundhaltungen, die für den sprachlichen Austausch von großer Bedeutung sind, werden integriert: aktives Zuhören, hermeneutisches vertieftes Verstehen einer Mitteilung, Umsetzung durch vielfältige Ausdrucksmöglichkeiten (Körper, Stimme u.a.), Lernen adäquater, situationsgerechter verbaler und nonverbaler Interaktion, Umgang mit Gefühlsebenen, Lernen als Transformationsprozess und Bewusstseinserweiterung, Aneignung integrativer Feedbackverfahren, Bildung gemeinsamer Erfahrungen durch Prozessanalyse und Wahrnehmen von interpersonellen und thematischen Zusammenhängen.

Der Ansatz PT bietet neue Möglichkeiten für solche Lernprozesse: Alltagserfahrungen, Erlebnisse, Konfliktmomente können damit aktiv angegangen werden, indem persönliche Fragmente aus dem Erfahrungsbereich von Einzelnen zu Lebensgeschichten in der Gruppe und dann zu gemeinsamem Austausch über geteilte Wirklichkeit werden. Darüber hinaus birgt die Methode PT nützliche Instrumente für die Mediation und zur Sensibilisierung für multikulturelle 
Situationen. Playback Theater dient dem Erlernen grundlegender zwischenmenschlicher Fähigkeiten und kommunikativer Grundhaltungen: einfühlendes Zuhören, Achtsamkeit auf sich und andere, Präsenz, Körperbewusstsein, Intuition, intellektuelle, affektive und emotionale Öffnung, adäquates Handeln, Selbst- und Fremdwahrnehmung, Rollenflexibilität, Selbstverantwortung, schöpferische Spontaneität.

\subsection{Playback Theater im universitären Lehrangebot}

Zur Veranschaulichung folgt das Setting einer Lehrveranstaltung an der Goethe-Universität Frankfurt am Main (Wintersemester 05/06). Sie wird als Theaterwerkstatt im Curriculum angekündigt. Adressaten sind RomanistikStudierende für Lehramt-, sowie Bachelor- und Masterstudiengänge. 14 Studierende nehmen teil.

Die Theaterwerkstatt ist in das Semesterangebot integriert. Die Lehrveranstaltung findet wöchentlich zweistündig in französischer Sprache statt (Niveau B2/C1 nach dem GER). Hauptziele sind Entwicklung von Sprachkompetenz durch globale Lernaktivierung, Abbau von Lern- und Sprechhemmungen durch Aufbau einer kommunikativen Atmosphäre, Förderung der Autonomie, erfahrungsbezogenes Lernen durch Handeln und durch Mitteilung biografischer, persönlicher Erlebnisse, Vermitteln innovativer Arbeitsansätze in der Lehre an der Hochschule.

In der ersten Sitzung werden nach einer kurzen Einführung die Grundformen der Arbeit veranschaulicht. Der Raum wird entsprechend umgebaut. In der Einstiegsphase, nach einer Reihe von Aufwärmübungen, werden die Themen der Sitzung rasch fokussiert. Die Gruppe steht im Kreis. Eine Studierende teilt ihre Stimmung zu Beginn des Semesters mit, das Gegenüber setzt dies durch die Form Sound \& Movement um. Sie sagt: „Die Atmosphäre hier gibt mir Sicherheit", das Gegenüber macht eine entsprechende Körperhaltung und Bewegung und begleitet mit Ton und Stimme. Das Grundprinzip des PT wird damit klar: einer erzählt, die anderen setzen es um und stellen die Essenz der Mitteilung in Kurzform dar.

Auch andere Themen werden durch diese verdichtete Darstellung deutlich: Verunsicherung in der Massenuniversität, mangelnde Orientierung, hohe Leistungsanforderungen und hoher Zeitaufwand.

Die Studierenden werden nun eingeladen, in Zweiergruppen ihre aktuellen Erfahrungen an der Universität auszutauschen. Durch diesen persönlicheren Kontakt wärmen sich die Kleingruppen an, sich für ihre real erfahrenen Geschichten zu öffnen. Danach finden in der Großgruppe im klassischen PT-Setting die ersten szenischen Umsetzungen statt. Zunächst wird unter meiner Leitung (als PT-Conductor) das Modell des PT-Interviews vermittelt. Ein Ort für die Bühne wird bestimmt, Darsteller stellen sich zur Verfügung. Die Zuschauer werden eingeladen, Erfahrungen zu Beginn des Semesters zu erzählen. Eine Studentin teilt eine frustrierende Erfahrung mit - kein Stuhl frei im Seminarraum, sie muss zwei Stunden lang in einem überfüllten Raum 
auf dem Boden sitzen. Die Aufmerksamkeit fällt schwer. Sie berichtet weiter, dass in einer Veranstaltung die verfügbaren Plätze per Los verteilt werden. Die Situation wird umgesetzt. Nach der Darstellung und dem Kommentar der Erzählerin („Hat die Darstellung die Essenz Ihrer Erfahrung erfasst?“), wird nach dem Echo dieser ersten Geschichte gefragt: Wer kennt Ähnliches? Welche Erfahrungen machen die Anderen?

Es folgen verbale Mitteilungen und szenische Umsetzungen. Nach einigen Szenen werden auch Polaritäten herausgearbeitet: Ambivalenzen dieser aktuellen Studierendengeneration werden deutlich, zwischen dem Wunsch effektiv in einem Kurzzeitstudium zu studieren und der Konfrontation mit nicht optimalen Studienbedingungen. Manche Polaritäten werden szenisch umgesetzt.

Am Ende der ersten Veranstaltung findet ein Feedback über den Verlauf und die Veranstaltungsform statt. Die Studierenden äußern hier ihr Erstaunen über die ungewöhnliche Arbeitsform und betonen die Lebendigkeit des entstandenen Austausches.

In der zweiten Sitzung wird an den Grundformen gearbeitet. Ein Kontinuum entsteht. Es wird verstärkt auf die Dynamik der Spiegelung eingegangen - ausgehend von Spiegelübungen. Die Themen der Szenen betreffen weiter das Studentenleben. Die Szenen sind Spiegel ihres Alltags: überfüllte Veranstaltungen einer Massenuniversität, die Schwierigkeit einander in Seminaren zuzuhören und zu verstehen, sich auf der Suche nach einem Buch in der Uni-Bibliothek zurechtfinden, aber auch die Freude, ein Fest in einem multikulturellen Studentenheim zu organisieren. Die Themen der zweiten Sitzung kreisen um Unsicherheit, Verunsicherung, aber auch um Neugier und Entdeckungslust.

Die Polarität des Alltags ist Fokus der dritten Sitzung: störende Momente und angenehme Erfahrungen. Parallel wird auf die szenische Umsetzung und ihre Dramaturgie mehr geachtet. Die Themen werden persönlicher. Es entstehen drei Szenen: eine Studentin aus Russland erzählt vom baldigen Wiedersehen mit ihrer Familie und ihren Verwandten in Novosibirsk - 7000 km entfernt - bei minus 20 Grad! Ein Student erklärt, wie er bei einer Juraprüfung durchgefallen ist; ein weiterer Student erzählt von seiner Verwirrung bei der Suche nach einem Seminarraum. Es wird auch nach den weiteren nicht dargestellten Themen gefragt: ein Student kam zu spät, der Zugverkehr wurde unterbrochen, da ein Reisender sich das Leben genommen hatte-dieser Student schwankte zwischen Wegschauen und Betroffenheit. Weiteres Thema: eine Vollversammlung gegen Einführung der Studiengebühren wird an der Uni vorbereitet - wer geht hin? Ein weiteres Thema: eine Studentin klagt über Kopfschmerzen, sie erzählt von ihrem Unwohlsein und teilt ihr Gefühl mit, hier an der Uni verloren zu sein. Im Austausch stellen wir Verbindungen zwischen den Themen der Einzelnen her.

In der vierten Sitzung wird verstärkt Rhythmus eingesetzt und Musikinstrumente werden eingeführt. Die Funktion der Musik im PT-Setting wird integriert: die Musik setzt u.a. den Rahmen (Anfang, dramatische Steigerung, Ende). Die Musik hilft auch der Gestaltung und der Darstellung der Szenen: dramaturgische Akzente und emotionale Färbung werden somit pointiert. 
Diese Art der nonverbalen Kommunikation schafft eine engere Verbindung der Studierenden untereinander. Spontane Impulse und Handlungen werden freigesetzt und eine Vertiefung der Themen findet statt: Konflikte im Alltag kommen zur Sprache. Eine Studentin erzählt, wie sie als Radfahrerin von einem unachtsamen Autofahrer fast verletzt worden wäre. Eine weitere Studentin erzählt vom Generationenkonflikt im überfüllten Hörsaal während einer Vorlesung in Erziehungswissenschaften: eine jüngere Studentin nimmt einem älteren Gasthörer seinen Sitzplatz weg, dieser zieht ihr wutschnaubend regelrecht die Hose herunter! 400 Studierende und der Professor stehen sprachlos da. Der Professor wechselt das Thema seiner Vorlesung und spricht nun von sich verbreitender Entfremdung an der Universität und in der Gesellschaft. Nach der szenischen Umsetzung folgen weitere Beiträge über Konkurrenz im Alltag oder auf dem Spielfeld bei einer Fußballmeisterschaft. Diese Erzählungen über negative Erfahrungen und zwischenmenschliche Disharmonie betonen gesellschaftliche Dissonanzen und Polaritäten. Die letzte Erzählung beleuchtet das Thema Musikkonzert im Freien im Sommer und Entspannung.

Ab der fünften Sitzung und in den folgenden Sitzungen bis zur Weihnachtspause 2005 werden weitere Grundformen der PT-Methode mit Musik und Requisiten (Verwendung von Tüchern) eingesetzt. Das Ritual eines klassischen Verlaufs wird auch bewusster und gezielter vermittelt. Die Themen der szenischen Umsetzung in dieser Phase betreffen sowohl den Universitätsalltag als auch persönlichere Erfahrungen im Alltag über Gelingen und Misslingen, zwischen Angst und Freude.

Die Studierenden öffnen sich zunehmend ästhetischer Erfahrung. Eine Musikstudentin bringt ihre Geige mit und übernimmt die Rolle der Musikerin im PT-Setting. Diese neue Dimension führt zu mehr Kreativität in der szenischen Gestaltung und beeinflusst auch die eingebrachten Themen. Eine Vielfalt der Themenwahl entsteht und der Wahrnehmung von roten Fäden und Zusammenhängen wird mehr Beachtung geschenkt. Aktuelle Themen wechseln mit früheren Erfahrungen. In einer Sitzung entstehen sehr unterschiedliche Geschichten: vom Tanzunterricht, in welchem die Schülerinnen einem autoritären Lehrer nicht zuhören und sich zunehmend selbständig machen; von einer stressigen multikulturellen Begegnung in einem überfüllten Wagen in der Pariser U-Bahn im heißen Hochsommer; von einer über gemeinsame Interessen entstandenen Freundschaft.

Eine tiefere verbindliche Kommunikation unter den anwesenden Kommilitonen entsteht in dieser Phase. Es ist auch eine Einladung, in einem wechselseitigen Prozess und Austausch Autor und Darsteller von persönlich erlebten Erfahrungen und Geschichten zu werden. Das PT-Setting schafft viel Vertrauen. Die Rollen sind klar verteilt. Die jeweils Erzählenden bleiben in der Rolle von verbal Mitteilenden auf einem ihnen zugewiesenen klaren Platz (sog. Erzählerstuhl), während die Darsteller auf der Bühne mit Tüchern als Requisiten in engem Kontakt mit einem Musiker stehen. Die subjektive Perspektive des Einzelnen wird von der Gruppe wahrgenommen, und das führt zu erhöhter gemeinsamer Aufmerksamkeit füreinander. Das Zusammenspiel wird von Sitzung zu Sitzung 
leichter, obwohl die Anforderungen sehr hoch sind: spontanes Spiel in der Fremdsprache. Hier lernen die Beteiligten alle verfügbaren nonverbalen Mittel vermehrt einzusetzen. Die Einzelnen erfahren auch von Sitzung zu Sitzung, dass sie oft gemeinsame Themen und entsprechende Gefühlslagen teilen.

In der zweiten Phase des Wintersemesters, ab Januar 2006, sollen sich die Studenten in die Dynamik der Rolle des PT-Conductors einfühlen und die Grundhaltung eines Interviews in Kleingruppen einüben und sich damit aneignen. Die Grundformen im Interview eines Erzählenden sind klar strukturiert und nehmen die Form eines Rituals an (Wann und Wo findet die Geschichte des Erzählenden statt? Wer sind die wichtigen Personen in der mitgeteilten Geschichte? Was passiert und wie endet die erzählte Geschichte?). Im Interview werden nach und nach die wichtigen Rollen verteilt und an die Darsteller weitergegeben: der Erzählende wird vom PT-Conductor eingeladen, jemanden auf der Bühne für seine Rolle auszuwählen, dann werden weitere Rollen an Darsteller verteilt. Nach und nach stehen die ausgewählten Darsteller in einer besonderen Position - sie treten einen Schritt vor (In-Between) und stellen sich somit dem jeweiligen Erzählenden zur Verfügung.

Diese ritualisierte Struktur macht die Identität der PT-Methode aus und unterscheidet sich damit vom Setting des Psychodramas oder vom Setting eines Forum-Theaters nach der Boal-Methode. Der Erzähler im Playback Theater wird nicht zum Protagonisten der eigenen Szene auf der Bühne: er bleibt in der Zuschauerrolle aus seiner besonderen Position (Erzählerstuhl und Stuhl des PT-Conductor befinden sich zwischen Bühne und Publikum). Nach der Erzählung einer Geschichte werden die Darsteller eingeladen, die Essenz der Szene in verdichteter Form wiederzugeben. Anders als im Forum-Theater wird während einer Darstellung vom Publikum nicht eingegriffen. Am Ende einer Darstellung wird im Austausch zwischen PT-Conductor und Erzähler festgestellt, ob die szenische Umsetzung die Essenz der Erfahrung des Erzählers getroffen hat. Bei starker Abweichung zum Erzählten wird dies thematisiert und es wird reflektiert, wie andere Möglichkeiten der szenischen Umsetzung entstehen und wie Erzähler wertgeschätzt werden können. Die PT-Methode stellt einen hohen Anspruch dar - eine ethische Grundhaltung wird vermittelt: sich in den Dienst der Anderen zu stellen!

Während die Studierenden zunehmend die Rolle des PT-Conductor übernehmen, findet zu diesem Zeitpunkt des Semesters auch eine Verlagerung in der Themenwahl statt. Eine Öffnung zu anderen Erfahrungen als im Universitätsalltag führt zur Darstellung von komplexen Situationen in multikulturellen Zusammenhängen. Was verbindet diese Erzählungen? Fehlkommunikation, Missverständnisse und die Kunst, damit umzugehen. Diese Themen sind Spiegelbild der Erfahrungen der Studierenden, die in Übungen zum PT-Conductor erleben, wie wichtig aktives Zuhören und gegenseitiges Verstehen in einer Fremdsprache sind. Einige Beispiele:

Im Nachhilfeunterricht Französisch zeigen die Schüler ihre negativen Einstellungen zur französischen Sprache, der Nachhilfelehrer ist gefordert, trotzdem Motivation zu fördern; eine Gruppe deutscher Reisender ist unterwegs 
in Frankreich, und wegen mangelnder Sprachkenntnisse erhalten die Reisenden nicht die Schlüssel zu einer Ferienwohnung; ein junges Au Pair-Mädchen in Quebec/Kanada kann sich nicht richtig verständigen und verpasst am Flughafen die Gastfamilie, die sie abholen will; ein Student erzählt von seiner multikulturellen Verwandtschaft (italienisch-deutscher Herkunft mit neuen Familienmitgliedern amerikanisch-tschechischer Herkunft) und wie wichtig es ist, sich jeweils Gehör zu verschaffen; eine Studentin erzählt, wie sie sich als Lehrerin in ihrer multikulturellen Schulklasse durchsetzen muss und was sie zunächst unternimmt, um von Schülern anderer Kulturen richtig anerkannt zu werden.

In der letzten Phase des Semesters findet eine intensivere Rückmeldung zum gesamten Verlauf statt. In Gruppengesprächen werden die Erfahrungen der Einzelnen zur Sprache gebracht. Übereinstimmend wird diese Veranstaltung als ein Kernstück zur innovativen Lehre in doppelter Hinsicht - an der Hochschule und im Fach Romanistik - betrachtet. Diese Phase der Rückmeldung der Studenten bedient sich zweier Formen, jeweils in der Zielsprache (Französisch). Ziel ist Anleitung zur Selbstreflexion:

- Eine individuelle zehnminütige mündliche Präsentation mit anschließendem Gruppengespräch;

- Das Erstellen eines schriftlichen Portfolios über die eigenen Lernwege während des Semesters mit subjektiver Einschätzung des Lernwertes (Entwicklung sprachlicher und kommunikativer Kompetenz, Sammeln und Beschreiben von persönlichen wachstumsorientierten Erfahrungen, Sprach- und Lernbiografie als Prozess).

Eine von der Goethe-Universität zentral durchgeführte Lehrveranstaltungsevaluation gibt folgende Hinweise/Eckpunkte aus der Rückmeldung der Studierenden:

Die Lehrveranstaltung hat folgende Stärken (Äußerungen der Studierenden):

- Man lernt die Sprache auf andere Art und Weise.

- Man lernt aktiv sich mündlich im Französischen zu verbessern.

- Die Arbeit wird in der Gruppe durch das Theaterspielen deutlich gefördert.

- Das Theaterspielen motiviert, das Angelernte zu vertiefen und anzuwenden.

- Man lernt spontan, das Wort zu ergreifen.

- Man lernt, sich mehr zu öffnen.

- Man lernt kreativer zu sein.

- Abwechslungsreiche Dynamik innerhalb der Gruppe. 
- In der Lehrveranstaltung herrscht ein konstruktives positives Klima.

- Sehr gute Inhaltsvermittlung.

- Das Tempo der Lehrveranstaltung ist angemessen.

- Die Veranstaltung ist klar strukturiert und es macht große Freude.

- Ich besuche regelmäßig die Veranstaltung.

\section{Anders Lernen / Anders Lehren}

Der Gemeinsame Europäische Referenzrahmen für Sprachen (GER) bietet einen neuen Ansatz in der Fremdsprachenvermittlung. Darin heißt es (http://www.coe.int/T/DG4/Portfolio/documents/Framework_EN.pdf): An action-oriented approach
A comprehensive, transparent and coherent frame of reference for lan- guage learning, teaching and assessment must relate to a very general view of language use and learning. The approach adopted here, gener- ally speaking, is an action-oriented one in so far as it views users and learners of a language primarily as 'social agents', i.e. members of soci- ety who have tasks (not exclusively language-related) to accomplish in a given set of circumstances, in a specific environment and within a partic- ular field of action. While acts of speech occur within language activities, these activities form part of a wider social context, which alone is able to give them their full meaning. We speak of 'tasks' in so far as the ac- tions are performed by one or more individuals strategically using their own specific competences to achieve a given result. The action-based approach therefore also takes into account the cognitive, emotional and volitional resources and the full range of abilities specific to and applied by the individual as a social agent.

Wie schon ausführlich beschrieben, bietet der Ansatz des Playback Theaters einen besonderen vorteilhaften Raum für den handlungsorientierten Ansatz des GER.

Playback Theater als Teil einer sich entwickelnden Relationellen Dramaturgie bietet einen prozessorientierten Rahmen, in welchem die Haltung der Achtsamkeit auf sich und Andere, die Selbst- und Fremdwahrnehmung und die Selbstreflexion besonders gefördert werden.

Dieser Prozess erlaubt das Schaffen eines privilegierten Raums, eines Ortes des Erzählens, wo die eigene Geschichte mitgeteilt wird und in Relation zu den Geschichten der anderen gesehen werden kann. Die Methode des Playback Theaters stellt hier den Rahmen für vielfältige neue innovative Entwicklungen in der Fremdsprachenausbildung dar: es handelt sich somit um eine offene Dialogform, in der Lernende $\mathrm{zu}$ aktiven Darstellern werden und sowohl Handelnde als auch Subjekte ihrer Lebensgeschichten sind. Aktiv Lernende 
werden zu Social Actors im Sinne von Jonathan Fox. Dies entspricht auch der Intention des GER.

Mit der aktuellen europäischen Entwicklung und dem Wandel in der Organisation universitärer Curricula (Modularisierung, Einrichtung von neuen europäischen Studiengängen) öffnen sich auch neue Möglichkeiten, innovative Arbeitsansätze wie die Playback-Theatermethode zu integrieren, um die Entwicklung demokratischer Partizipation über sprachliche, kulturelle und ästhetische Erziehung zu fördern (vgl. Feldhendler 2007).

\section{Auf dem Wege zu einer „Pädagogik des Seins“}

Dufeus Konzept einer "Pädagogik des Seins"(1983) im Fremdsprachenunterricht ist in Verbindung zu sehen mit gesellschaftlichen Entwicklungen im Laufe der 70er Jahre, die auch innovativ in den Bereich Unterrichtmethodik hinein wirkten. Diese Pädagogik ließe sich auch als Psychodrama-Pädagogik verstehen. Ihre Hauptmerkmale sind folgende:

- Die Lernenden werden als Individuen und Subjekte betrachtet;

- Die Lernenden sind Teilnehmer einer Lerngruppe: es findet ein personenund gruppenorientierter Unterricht statt;

- Die Teilnehmer werden in ihrer Gesamtheit (Körper, Gefühlsleben, Intellekt) berücksichtigt;

- Der Spracherwerb ist weniger auf die Speicherung von Inhalten gerichtet als auf die Entwicklung von Fähigkeiten, Fertigkeiten und Haltungen, die den Erwerb der Fremdsprache fördern;

- Die erworbenen Fähigkeiten tragen zur besseren Wahrnehmung des Selbst, der anderen Teilnehmer und der Umwelt bei;

- Die Entwicklung dieser Fähigkeiten als kommunikative Kompetenz ermöglicht dem Teilnehmer, sich als Person auszudrücken und sich zu situieren;

- Die Sprache wird nicht mechanisch gelernt (wie in Strukturübungen), sondern in direktem Kontakt mit Bezugspersonen erlebt, erfahren und erworben;

- Die Erlebnisse der Teilnehmer werden einbezogen. Der Teilnehmer kann sich mit seiner eigenen Identität, mit der Wahrnehmung seiner Realität, mit dem Ausdruck seiner Phantasie, als Person einbringen

- Die Sprache ist Mittel zur zwischenmenschlichen Kommunikation;

- Das inhaltliche Programm des Spracherwerbs entsteht in Anwesenheit der Teilnehmer und gestaltet sich nach ihren Ausdrucksbedürfnissen; 
- Die Lernschritte richten sich nach interaktiven, relationellen Komponenten;

- Der Irrtum ist ein wesentlicher Bestandteil des Erwerbsprozesses;

- Die Lehrerrolle und die pädagogische Beziehung ändern sich: die hierarchische Beziehung wird durch eine empathische Beziehung ersetzt.

Bestimmte Arbeitsbedingungen für die Pädagogik des Seins sind notwendig:

- Eine Raumgestaltung, die Beweglichkeit in der Gruppe erlaubt;

- Zeit und Raum für Kleingruppenarbeit;

- Offenes Inhaltsangebot;

- Feedback-Verfahren in der Gruppe;

- Eine breite und gründliche Lehrerausbildung und kontinuierliche Fortbildung.

\section{Bibliographie}

Deutscher Fachverband für Psychodrama e.V.:

http://www.psychodrama-deutschland.de/

Psychodramaturgie: http://www.psychodramaturgie.de/

Centre for Playback Theatre (PT-Resources):

http: //www.playbackcentre.org/

International Theatre of The Oppressed:

http: //ww . theatreoftheoppressed.org/en/

Gemeinsamer Europäischer Referenzrahmen für Sprachen:

http://www.coe.int/T/DG4/Portfolio/documents/Framework_EN.pdf

(2008): Interaktives Theater an der Hochschule: Playback Theater und

Spiegelbühne. In: Wildt, Beatrix; Hentschel, Ingrid; Wildt, Johannes (eds.):

Theater in der Lehre. Wien, Zürich, Münster: LIT Verlag, 221-236

(2008): Playback Theater und Biografieforschung. In: Dauber, Heinrich (eds.):

Wo Geschichten sich begegnen - Gathering voices. Dokumentation der Verleihung der Ehrendoktorwürde an Jonathan Fox in Anerkennung seiner wissenschaftlich-künstlerischen Leistungen auf dem Gebiet der Theaterpädagogik. Kasseler Beiträger zur Erziehungswissenschaft, Band 3. Kassel: Kassel University Press.

http://www.playbackschool.org/articles/Feldhendler.Playback\%20Theater\%20und\%20Biografieforsch

(2008): Mise en scène de récits biographiques: reliance et médiation transculturelle. In: Alao, George et al. (eds.): Grandes et petites langues. Pour une didactique du plurilinguisme et du pluriculturalisme. Transversales 24, Langues, sociétés, cultures et apprentissages. Bern: Peter Lang, 227-238 
(2007): Médiations sociales et théâtre-récit. In: Delory, Christine; Biarnès, Jean (eds.): Insertion, Biographisation, Education. L'orientation scolaire et professionnelle $36 / 1,45-58$

(2007): Playback Theatre: A Method for Intercultural Dialogue. In: Scenario 2, 2007. http://epu.ucc.ie/scenario/2007/02/feldhendler/04 (2006): La vie mise en scène, théâtre et récit. In : Molinié, Muriel (eds.): Biographie langagière et apprentissage plurilingue. In: Le français dans le monde, Recherches et applications 39, 155-168

(2005): Théâtre en miroirs, l'histoire de vie mise en scène. Paris: Téraèdre

(2004): Playback Theater. In: Koch, Gerd ; Roth, Sieglinde; Vaßen, Florian;

Wrentschur, Michael (eds.): Theaterarbeit in sozialen Feldern.

Frankfurt/Main: Brandes \& Apsel, 147-152

(2002): Apprendre et enseigner autrement: une pédagogie de la relation. In: Grenzgänge 9. Leipzig und Frankfurt/Main: Leipziger Universitätsverlag 17, 75-87

(2000): Psychodramatische und dramaturgische Arbeitsformen in der Sprachvermittlung. In: Wittinger, Thomas (ed.): Psychodrama in der Bildungsarbeit. Mainz: Matthias Grünewald, 11-29

(2000): Apprendre avec tous les sens: Pratiques relationnelles et dramaturgiques. In: Fremdsprachenunterricht - Themenheft Ganzheitliches Lernen mit allen Sinnen. Berlin: Pädagogischer Zeitschriftenverlag 4, 290-294 (1999): La dramaturgie relationnelle. In: Le français dans le mondeRecherches et Applications, 125-133

(1996) Inszenierung interkultureller Selbst-/Fremdbilder in der Fremdsprachenausbildung. In: Ambos, Erwin (eds.): Interkulturelle Dimension der Fremdsprachenkompetenz. Bochum: AKS Verlag, 257-268 (1993): Augusto Boal and Jacob L. Moreno - Theatre and Therapy. In: Schutzman, Mady; Cohen-Cruz, Jan (eds.): Playing Boal. London: Routledge, 87-109

(1993): Enacting Life! Proposal for a Relational Dramaturgy for Teaching and Learning a Foreign Language. In: Schewe, Manfred; Shaw Peter (eds.): Towards Drama as a Method in the Foreign Language Classroom. Frankfurt/Main: Peter Lang, 171-191

(1992): Psychodrama und Theater der Unterdrückten. Frankfurt/Main: Nold, 2. Auflage

(1991): Aus dem Leben gegriffen! In: Ruping, Bernd (ed.): Gebraucht das Theater. Die Vorschläge Augusto Boals: Erfahrungen, Varianten, Kritik.

Remscheid: Schriftenreihe der Bundesvereinigung Kulturelle Jugendbildung 17, 160-170

(1991): Das Leben in Szene setzen! Ansätze für eine fremdsprachliche Dramaturgie. In: Die Neueren Sprachen 2, 137-153

(1990): Dramaturgie et interculturel. In: Le français dans le monde 234, 50-60 
(1990) Aus dem Leben gegriffen. Szenische Darstellung von Zeitungsnachrichten als Unterrichtseinheit. In: AZ-Journal 2. München: Goethe Institut, 109-112 (1989): Das lebendige Zeitungstheater - Teilnehmeraktivierung im Fremdsprachenunterricht durch relationelle und dramaturgische Arbeitsformen. In: Addison, Anthony; Vogel, Klaus (eds.): Gesprochene Fremdsprache. Bochum: AKS Verlag, 119-140

(1988): Le théâtre journal vivant. In: Le français dans le monde 220, 56-61 (1983): Expression Dramaturgique. In: Le français dans le monde 176, 45-51 Online-Resourcen:

Ausgewählte Publikationen von Daniel Feldhendler zum Thema: Das Leben in Szene setzen - Wege zu einer Dramaturgie Relationnelle: 